\title{
Bayesian Analysis of Ellipsometry Measurements
}

Udo v. Toussaint, Thomas Schwarz-Selinger and Christian Hopf

Max-Planck-Institut für Plasmaphysik, EURATOM Association, Boltzmannstr. 2, D-85748 Garching, Germany

\begin{abstract}
Ellipsometry is a unique technique of great sensitivity for in situ non-destructive characterization of surfaces utilizing the change in the state of polarization of a light-wave. It is extensively used in the semi-conductor industry. To relate ellipsometric measurements to surface properties (as eg layer thickness changes in the range of nm or chemical composition), Bayesian probability theory is used. The parameter estimation process is complicated by the incomplete phase information of the measured data. Examples of 3-D surface reconstructions of samples after ion bombardment demonstrate the tremendous information gain due to the Bayesian analysis.

keywords: Ellipsometry, Surface Analysis, Bayesian Inference, Parameter Estimation.
\end{abstract}

PACS numbers: 02.50Tt;78.20.-e;78.40.-q;78.68.+m;81.70Fy

Published in AIP Conference Proceedings: Received 10 July 2006

Accepted for publication 15 September 2006

AIP Conference Proceedings Vol.872 (2006)

p. $272-279$

\section{INTRODUCTION}

Ellipsometry is an experimental technique for the determination of the optical properties of thin films. It measures the change in the state of polarization upon reflection of a collimated light beam at a sample surface. For the case of a sufficiently transparent thin film on a substrate, the change in polarization carries information of the complex refractive indices $(\hat{n}=n-i \kappa, \mathrm{n}=$ refractive index, $\kappa=$ extinction coefficient) of both, the substrate and the thin film, and the film thickness. Because changes of the phase of light upon reflection can be measured with a sensitivity below $0.01^{\circ}$, sub-monolayer sensitivity to changes of film thickness or of $n, \kappa$ can be achieved $[2,4]$. The complex dependence of the measured physical quantity on the model parameters in combination with lacking phase information due to phase wrapping poses a challenging parameter estimation problem.

\section{ELLIPSOMETRY}

Ellipsometry has been used for decades as a standard ex-situ tool in semiconductor industry to measure the thickness of $\mathrm{SiO}_{2}$. In this special application the optical properties of the system are known with the thickness of $\mathrm{SiO}_{2}$ as the only unknown parameter. Here we tackle the generic case with limited knowledge about the optical properties and the (multi-)layer thicknesses. Ellipsometry is a monolayer-sensitive technique that can be used for real-time process control in such adverse environments as plasma, ion-beam or chemical vapor deposition. Here most of the standard surface science methods, involving low-energy electrons, fail. Therefore in-situ ellipsometry becomes more and more popular.

a. Setup The experimental equipment is rather simple as depicted in fig. 1. It consists of a light source, two polarizing elements, where one is acting as polarizer and one as analyzer (one of them is rotating) and the detector. The monochromatic light of a laser is linearly polarized in the first polarizer. The polarization of the reflected beam is altered by the optical properties of the sample and detected measuring the light intensity after the beam passed a second (rotating) polarizer. A comprehensive overview over the various techniques used for ellipsometry is given in [1].

b. Ellipsometric angles The time- and space-dependent vector of the electric field of planar, monochromatic light propagation in z-direction is given by

$$
\vec{E}(z, t)=\left(\begin{array}{c}
\hat{E}_{x} \cdot e^{i\left(\omega t-k z+\delta_{x}\right)} \\
\hat{E}_{y} \cdot e^{i\left(\omega t-k z+\delta_{y}\right)} \\
0
\end{array}\right) .
$$

In the following it is sufficient to consider only the amplitude and phase of the vector components of the electric field. This representation is known as Jones-vector[1]

$$
\vec{E}=\left(\begin{array}{c}
E_{x} \\
E_{y}
\end{array}\right)=\left(\begin{array}{c}
\hat{E}_{x} e^{i \delta_{x}} \\
\hat{E}_{y} e^{i \delta_{y}}
\end{array}\right) .
$$




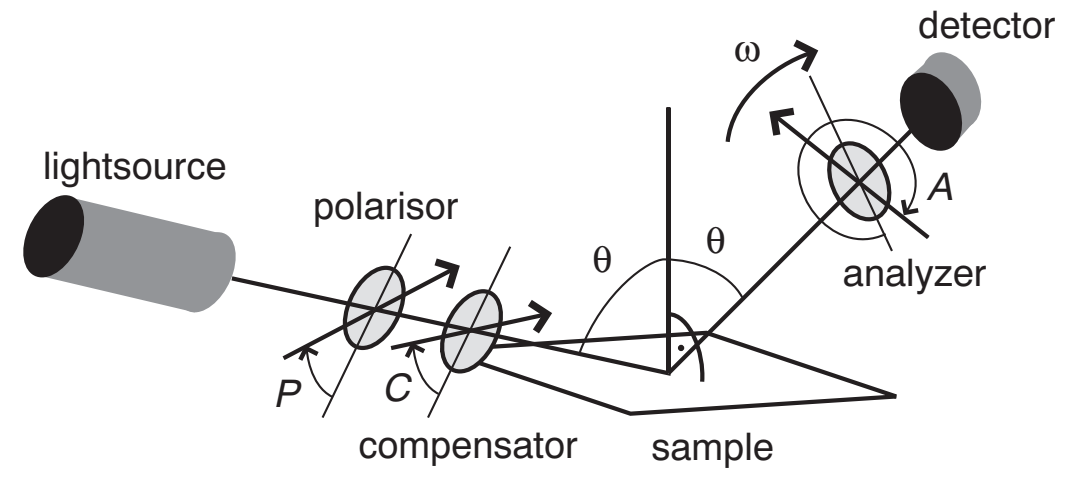

FIG. 1: The ellipsometer arrangement with a polarizing section that consists of a linear polarizer $P$ and a compensator $C$, and an analyzing section that consists of a linear analyzer $A$ and the photodetector.

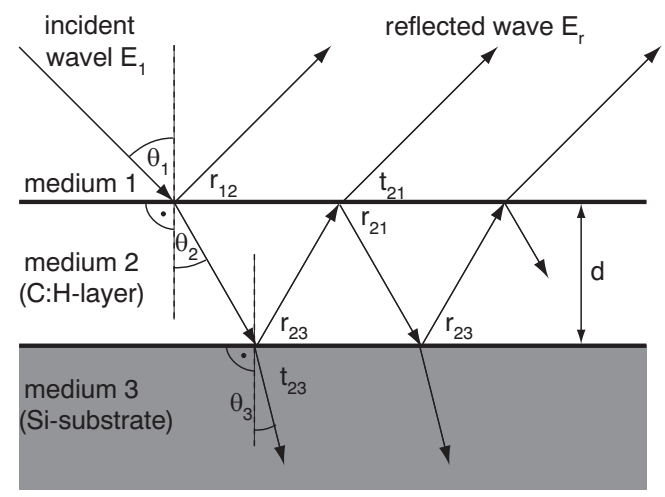

FIG. 2: Simplified reflection model of a two-layer system (a thin a-CH layer on top of a silicon substrate)

The measured quantity $\rho$ is the ratio of the complex reflectivities, $r_{\mathrm{p}}$ and $r_{\mathrm{s}}$, for pure $\mathrm{p}$ - and s-polarization states. $\mathrm{p}$ and s refer to the directions of polarization parallel (p) and perpendicular(s) to the plane of incidence.

$$
\rho=\frac{r_{\mathrm{p}}}{r_{\mathrm{s}}}=\frac{E_{\mathrm{p}}^{\text {out }} / E_{\mathrm{p}}^{\text {in }}}{E_{\mathrm{s}}^{\text {out }} / E_{\mathrm{s}}^{\text {in }}}
$$

It is common practice to use the ellipsometric angles $\Delta$ and $\Psi$ instead of the complex quantity $\rho$. The relation is given by

$$
\rho=\tan \Psi e^{i \Delta} \quad \text { with } \quad \Psi \in\left[0^{\circ}, 90^{\circ}\right], \Delta \in\left[0^{\circ}, 360^{\circ}\right]
$$

\section{Model of the optical system}

The relation between the ellipsometric angles $\Delta$ and $\Psi$ and the sample parameters is given by the effective reflection and transmission properties of the sample. These properties can be expressed by Fresnel coefficients for an interface between two layers [3]:

$$
\begin{aligned}
& r_{\mathrm{p}, 12}=\frac{E_{\mathrm{r}, \mathrm{p}}}{E_{0, \mathrm{p}}}=\frac{\hat{n}_{2} \cos \theta_{1}-\hat{n}_{1} \cos \theta_{2}}{\hat{n}_{2} \cos \theta_{1}+\hat{n}_{1} \cos \theta_{2}} \\
& r_{\mathrm{s}, 12}=\frac{E_{\mathrm{r}, \mathrm{s}}}{E_{0, \mathrm{~s}}}=\frac{\hat{n}_{1} \cos \theta_{1}-\hat{n}_{2} \cos \theta_{2}}{\hat{n}_{1} \cos \theta_{1}+\hat{n}_{2} \cos \theta_{2}} \\
& t_{\mathrm{p}, 12}=\frac{E_{\mathrm{t}, \mathrm{p}}}{E_{0, \mathrm{p}}}=\frac{2 \hat{n}_{1} \cos \theta_{1}}{\hat{n}_{2} \cos \theta_{1}+\hat{n}_{1} \cos \theta_{2}} \\
& t_{\mathrm{s}, 12}=\frac{E_{\mathrm{t}, \mathrm{s}}}{E_{0, \mathrm{~s}}}=\frac{2 \hat{n}_{1} \cos \theta_{1}}{\hat{n}_{1} \cos \theta_{1}+\hat{n}_{2} \cos \theta_{2}}
\end{aligned}
$$




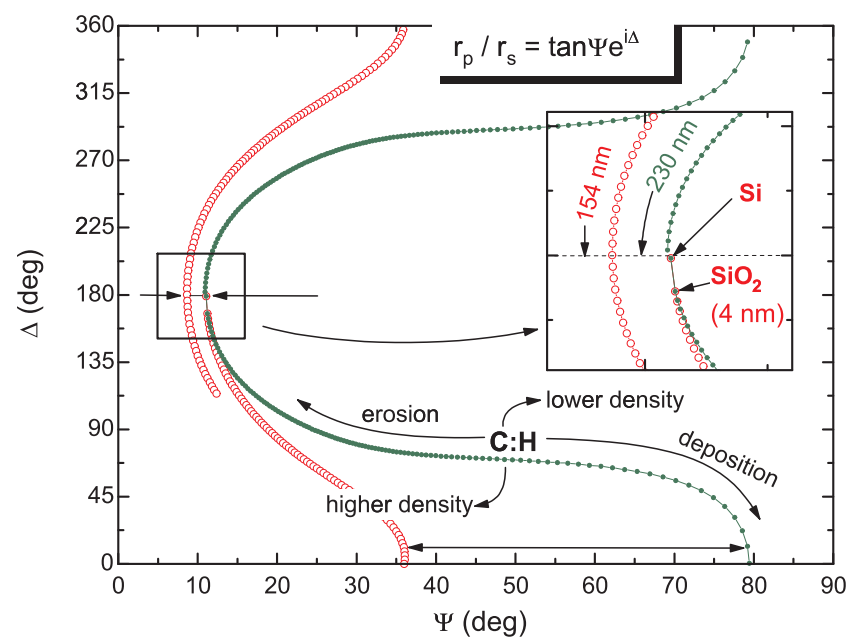

FIG. 3: Fig. 3 shows modeling results for 2 different films with homogeneous properties grown on a silicon substrate. The curves shown, calculated from Fresnel's equations, are representative of experimental results for soft, polymer-like (case A) and hard, $a-C: H$ films (case B). In the following these curves are designated psi-delta trajectories. They all start around psi $=11^{\circ}$ and delta $=16 \%$, which represent the values for a thin native $\mathrm{SiO}_{2}$ layer on $\mathrm{Si}$.

$\mathrm{p}$ and s refer to the directions of polarization parallel (p) and perpendicular (s) to the plane of incidence. Snell's law relates the corresponding reflection angles by

$$
n_{1} \sin \theta_{1}=n_{2} \sin \theta_{2} .
$$

A layered system as displayed in fig. 2, requires the coherent superposition of the individual rays. For this example the reflection coefficient is given by

$$
r_{\text {layer }}=r_{01}+t_{01} t_{10} \sum_{k=0}^{\infty} r_{12}^{k+1} r_{10}^{k} a^{k+1},
$$

taking into account the phase by

$$
a=\exp \left(-2 i \beta_{\text {phase }}\right) \quad \text { with } \quad \beta_{\text {phase }}=\frac{2 \pi}{\lambda} \cdot \hat{n}_{1} d \cos \theta_{1} .
$$

Using Eq. (5) and summing the geometric series in Eq. (7) finally yields

$$
r_{\text {layer }}=\frac{r_{01}+r_{12} \exp \left(-2 i \beta_{\text {phase }}\right)}{1+r_{01} r_{12} \exp \left(-2 i \beta_{\text {phase }}\right)} \text {. }
$$

For multi-layer systems the more efficient, although less obvious, matrix-based algorithm of Jones is used ([1], ch. 4.6).

In fig. 3 an example of the complex interaction of the different model parameters is given. Shown are modeling results as trajectories in the psi-delta plane for homogeneous $\mathrm{C}: \mathrm{H}$ films on silicon for two different sets of optical constants at an angle of incidence of $70^{\circ}$. In case A (solid circles): $\hat{n}=n-i \kappa=1.57-i 0.001$; case $\mathrm{B}$ (open circles): $\hat{n}=2.1-i 0.04$. The starting point for the model is in both cases the silicon single-crystal surface with a native 4 -nm thick $\mathrm{SiO}_{2}$ adlayer (see enlarged inset). The total film thickness for one complete revolution in the psi-delta plane (delta reaches again $180^{\circ}$, dashed horizontal line in the inset) is $230 \mathrm{~nm}$ in case A and $154 \mathrm{~nm}$ in case $\mathrm{B}$. The difference in thickness between consecutive model points is constant at $1.0 \mathrm{~nm}$. Case A is representative for polymer-like, case B for hard C:H films. Further shown is a compass card indicating the directions into which the optical answer of the system shifts if various modeling parameters are changed.

\section{EXPERIMENT}

It was suggested to probe reactive particle beams by eroding plasma deposited a-C:H films and measuring the modified film thickness by ellipsometry [5]. Additionally the easily accessible refractive index of plasma deposited a$\mathrm{C}: \mathrm{H}$ films is strongly correlated with physical quantities like density, carbon content or hydrogen content [6]. To obtain 


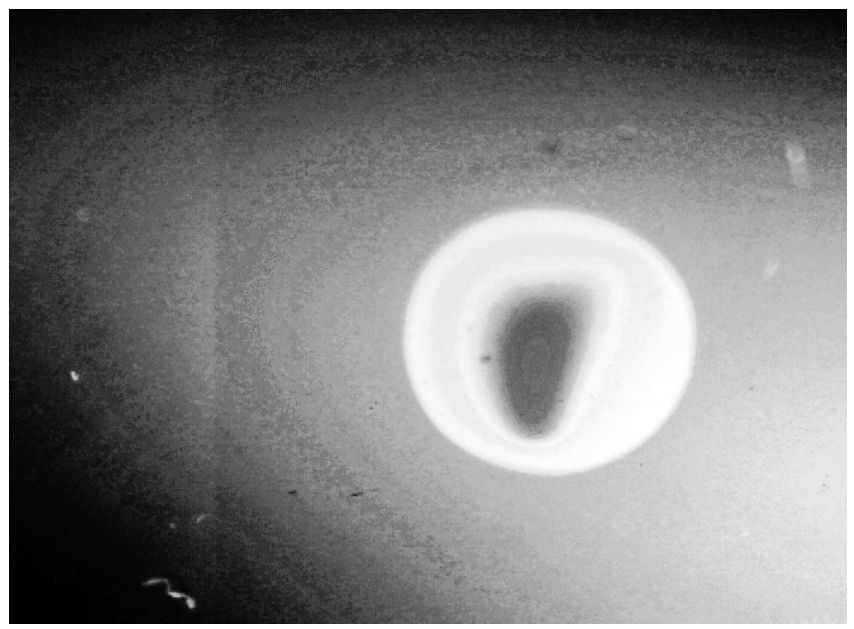

FIG. 4: Image of an amorphous C:H-layer deposited on a silicon substrate after bombardment with 1 keV deuterium ions. The intensity distribution of the beam spot is reflected in the color variations of the sample from green to red (here only the corresponding (digitally enhanced) gray scale image is shown).

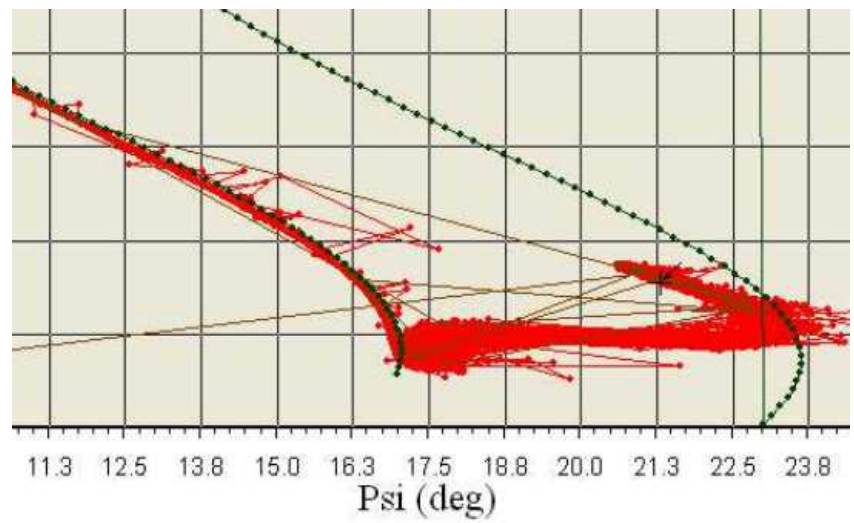

FIG. 5: The medium gray data cloud represents the measured data points plotted in $\Delta-\Psi$-representation. For comparison purposes the expected functional relationship for an undisturbed optical system of varying thickness is also displayed (black lines starting near the upper left corner). The discrepancy is a clear indication of the impact of the bombardment on the optical constants.

information about ion beam intensity and ion beam shape of a dual-source ion beam experiment at the accelerator of the IPP an amorphous C:H-layer was exposed to an $1 \mathrm{keV}$ deuterium ion beam. An image of the sample after exposure is given in fig. 4. The a-C:H layer is undisturbed outside the circular bounded beam spot (the beam spot is shaped by a circular aperture) as the homogenous gray scale indicates. In the center of the beam spot the intensity distribution of the deuterium beam is reflected in the a colored pattern (visible as brightness variations in the grayscale image). It seemingly deviates from the intended homogenous distribution. This (erosion) profile was subsequently analyzed by ellipsometry. The device used in this study is a single wavelength ex-situ rotating analyzer ellipsometer using a He-Ne laser at $632 \mathrm{~nm}$ (Jobin Yvon PZ 2000). The angle of incidence was $70^{\circ}$ and the polarizer was set to $45^{\circ}$. The laser spot size on the sample is 10 by 30 microns in size. A rectangular grid of 100 by 100 points has been sampled and the ellipsometric angles have been recorded at each point. The measured angles are plotted in fig 5 together with modeling results using an optical model valid for the un-exposed sample. In this simulation the optical constants (reflection and absorption coefficients) have been kept constant and only the thickness of the a-C:H-layer has been varied. The large number of data points in disagreement with the modeling results indicates that the a-C:H layer has been modified by the energetic ion beam. Therefore not only the thickness of the system has to be taken into account but also the change of the complex refraction indices of the layers. 


\section{BAYESIAN ANALYSIS}

For the case at hand the ellipsometry problem for a single point is severely under-determined. Every analyzed point provides the two ellipsometric angles only. On the other hand the number of parameters to be estimated is given by $3 N$, with $N=$ number of layers. Therefore, in our application $(N \geq 2)$ the ellipsometry problem is severely under-determined. Each layer $i=1 \cdots N$ is parameterized by three parameters: thickness $t_{i}$, refractive index $n_{i}$ and extinction $\kappa_{i}$. The likelihood function for the problem with measurement uncertainties $\sigma_{\Delta}$ and $\sigma_{\Psi}$ for data point $j$ reads:

$$
p\left(\Delta_{j}, \Psi_{j} \mid \vec{t}_{j}, \vec{n}_{j}, \vec{\kappa}_{j}, \sigma_{\Delta}, \sigma_{\Psi}, I\right) \propto \exp \left\{-\frac{\left(\Delta_{j}-f\left(\vec{t}_{j}, \vec{n}_{j}, \vec{\kappa}_{j}\right)\right)^{2}}{2 \sigma_{\Delta}^{2}}-\frac{\left(\Psi_{j}-g\left(\vec{t}_{j}, \vec{n}_{j}, \vec{\kappa}_{j}\right)\right)^{2}}{2 \sigma_{\Psi}^{2}}\right\}
$$

where $f\left(\vec{t}_{j}, \vec{n}_{j}, \vec{\kappa}_{j}\right)$ and $g\left(\vec{t}_{j}, \vec{n}_{j}, \vec{\kappa}_{j}\right)$ denote the models for $\Delta$ and $\Psi$ respectively. The posterior distribution of the parameters is obtained from Bayes theorem as

$$
p\left(\vec{t}_{j}, \vec{n}_{j}, \vec{\kappa}_{j} \mid \Delta_{j}, \Psi_{j}, \sigma_{\Delta}, \sigma_{\Psi}, I\right) \propto p\left(\Delta_{j}, \Psi_{j} \mid \vec{t}_{j}, \vec{n}_{j}, \vec{\kappa}_{j}, \sigma_{\Delta}, \sigma_{\Psi}, I\right) p\left(\vec{t}_{j}, \vec{n}_{j}, \vec{\kappa}_{j} \mid I\right) .
$$

The available knowledge of the experimentalists about the parameter values prior to the experiment is encoded in proper, bounded and uniform priors

$$
p\left(\vec{t}_{j}, \vec{n}_{j}, \vec{\kappa}_{j} \mid I\right)=p\left(\vec{t}_{j} \mid I\right) p\left(\vec{n}_{j} \mid I\right) p\left(\vec{\kappa}_{j} \mid I\right),
$$

assuming independence of the parameters (which neglects the Kramers-Kronig relationship). Making the layered structure explicit we can write $\left(x=\left\{t_{i j}, n_{i j}, \kappa_{i j}\right\}\right)$

$$
p(x \mid I)=\frac{1}{x_{\text {high }}-x_{\text {low }}}, \text { for } x \in\left[x_{\text {low }} \cdots x_{\text {high }}\right] .
$$

The first experiences with this evaluation were disappointing: Virtually dozens of solutions with different parameter vectors yielded the same quality of the fit and were indistinguishable. The solution space was much larger than the practitioners had anticipated. To overcome this difficulty the different length scales of thickness changes and changes of the optical properties had to be exploited. A user-defined multi-scale approach has been implemented which takes into account that in most cases the thickness variations are on a smaller length scale than changes of the optical properties. Essentially the user has to provide the information on which length scale he expects the optical properties of the sample to be constant. With this specification more data points can be used for the estimation of the parameters reducing the intrinsic variability of the system. This approach worked quite well. More elaborate attempts (eg. imposing smoothness by $2 \mathrm{~d}$-splines) were not successful either due to extensive computing time requirements or because they got easily trapped in the large number of local minima.

\section{RESULTS}

The multi-scale approach has been applied to the sample shown in fig. 4 before and after exposure to the $1 \mathrm{keV}$ deuterium ion beam using a four layer system. The initial optical properties of the sample corresponded to a hydrogenrich, so called soft a-C:H-layer with $\hat{n}=n-i \kappa=1.57-i 0.001$ and an average thickness of $t=230 \mathrm{~nm}$. Using those parameters for the depth estimation after exposure failed in the area of the beam spot. Fig. 5 reveals the reason for this failure. The measured data points are plotted in the $\Delta-\Psi$-plane. Additionally the computed ellipsometric angles for the undisturbed optical system of a soft a-C:H layer with thickness changes in steps of $1 \mathrm{~nm}$ are given as black dots connected by a black line. Within the measurement uncertainty all measured data points of an a-C:H layer should coincide with the model values. Nevertheless the measured data points do not lie on the expected path in the $\Delta-\Psi$-plane for reasonable and constant values of extinction and absorption coefficients. This indicates that the deuterium atoms with the relatively high energy of $1 \mathrm{keV}$ did not only erode the a-C:H layer but did modify also its optical constants. The energetic particles released hydrogen from the layer, transforming it into a hard a-C:H layer with a much higher refraction coefficient of approximately $n \approx 2.1$. The estimated depth profile (mode values) of the multi-layer-system is shown in fig. 6. Outside the beam spot the previous thickness of $t=230 \mathrm{~nm}$ is reconstructed and also the optical properties of a soft a-C:H layer. Inside the irradiated area the optical properties change within the outer $30 \%$ of the radius of the beam spot to those of a hard a-C:H layer. At the same time the thickness of the layer is reduced to $170 \mathrm{~nm}$ in the center. This information obtained by the bayesian analysis of the ellipsometry data is now used to optimize the beam optics of the dual-beam experiment at IPP. 


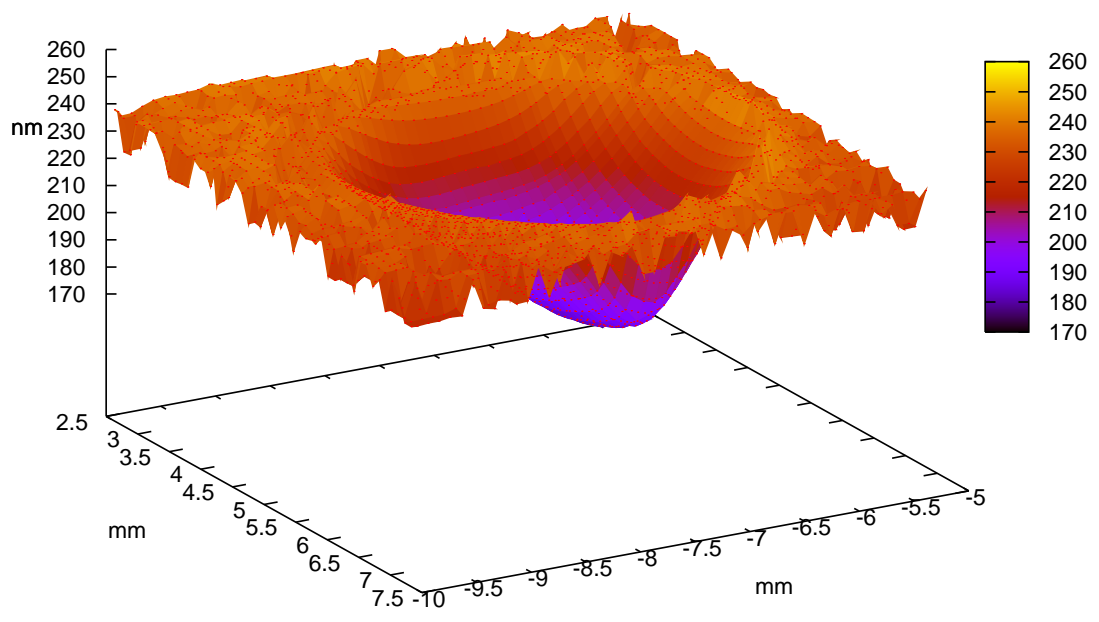

FIG. 6: Depth profile obtained by the Bayesian evaluation. The erosion dominated area in the center of the beam spot is nicely reconstructed, also the thickness of the deposited a-C:H-layer outside of the beam spot is in excellent agreement with the expected thickness of $t=230 \mathrm{~nm}$.

\section{CONCLUSION}

We have shown that the Bayesian analysis of ellipsometry measurements overcomes limitations present in the conventional way of evaluating these experiments. This extends the applicability of ellipsometry to situations where both the layer thicknesses and the optical constants are variables and also adds ellipsometry to the number of surface analysis techniques which have been analyzed from a Bayesian point of view [7]. Regarding the feedback of many people working in the surface physics area it seems that there is an increasing demand of joint evaluation of the different analysis techniques to overcome the limitations of the individual techniques - a promising area for Bayesians.

[1] R. Assam and N. Bahara, Ellipsometry and polarized Light, North Holland Publ., Amsterdam (1988).

[2] R. W. Collins, J. Vac. Sci. Technol. A 7, 2874 (1989).

[3] E. Hecht, Optics, Addison-Wesley, 1987.

[4] A. von Keudell and T. Schwarz-Selinger and W. Jacob, Simultaneous Interaction of Methyl Radicals and Atomic Hydrogen with Amorphous Hydrogenated Carbon Films, J. Appl. Phys. 89, 2979 (2001).

[5] T. Schwarz-Selinger and A. von Keudell and W. Jacob, Novel method for absolute quantification of the flux and angular distribution of a radical beam source for atomic hydrogen, J. Vac. Sci. Technol. A 18, 995 (2000).

[6] T. Schwarz-Selinger and A. von Keudell and W. Jacob, Plasma chemical vapor deposition of hydrocarbon films: The influence of hydrocarbon source gas on the film properties, J. Appl. Phys. 86, 3988 (1999).

[7] U. von Toussaint and V. Dose, Bayesian Analysis of Surface Diagnostics, Applied Physics A 82,403-413 (2006). 